\title{
Morphological change of PTFE by ion micro beam irradiation
}

\author{
Akane Kitamura, Takahiro Satoh, Masashi Koka, Tomihiro Kamiya, \\ Tomohiro Kobayashi* \\ Department of Advanced Radiation Technology, Takasaki Advanced Radiation Research Institute, \\ Japan Atomic Energy Agency (JAEA), \\ 1233 Watanuki-machi, Takasaki, Gunma 370-1292, Japan, \\ Tel: +81-27-346-9350, Fax: +81-27-346-9690,E-mail:ogawa.akane@jaea.go.jp \\ * RIKEN, 2-1 Hirosawa, Wako-shi, Saitama 350-0198, Japan
}

\begin{abstract}
Polytetrafluoroethylene (PTFE) is used in various industrial applications because of its desirable physical and chemical properties. PTFE is chemically inert under most conditions, which means that there are few suitable microfabrication techniques available, and these are top-down processes. We report the fabrication of microstructures at desired spots on the PTFE surface using a $3 \mathrm{MeV}$ focused proton microbeam with no patterning masks. The beam size on the surface was about $1 \mu \mathrm{m}$, and the surfaces were observed by scanning electron microscopy. When the 100- $\mu \mathrm{m}$-thick PTFE sample was circularly scanned, the irradiated areas expanded and were raised above the original surface level. When the thickness of the PTFE was increased, the edge of the structure became rough. Spiral scanning from the center of the circle in the $500-\mu \mathrm{m}$-thick sample formed a $250-\mu \mathrm{m}$-tall cone. These microstructures were created by the volume expansion along the ion trajectories in the PTFE. The structures were formed through a bottom-up process and were dependent on the proton beam and the sample thickness.
\end{abstract}

Key words: ion micro beam, PTFE, microfabrication, surface morphology

\section{INTRODUCTION}

Polytetrafluoroethylene (PTFE) $\left(-\mathrm{CF}_{2}\right)_{n}$ is an important material for biochemical and medical tools, and microelectromechanical systems (MEMS), because of its high chemical stability, electrical resistivity, and weathering resistance. Synchrotron radiation (SR) $[1,2]$ and ion beam irradiation [2-5] are effective for fabricating microstructures on the surface of PTFE, because it is extremely sensitive to ionizing radiation. SR has previously been used to fabricate microstructures by using both masks and sample heating [3]. Because these methods etch the irradiated area, the structures are formed at the etched surface which is damaged by irradiation. These methods etch the irradiated area, thus it is not possible to create microstructures on the original PTFE surface. Recently, we have reported that fine micro-protrusions could be formed on PTFE surfaces by using an $80 \mathrm{keV} \mathrm{N}_{2}{ }^{+}$ion beam [3-5]. The protrusions were formed through a top-down process which increased the size and the number of micropores in the surface. In this process, the level of the top surface was declined during irradiation (i.e. top-down process, which creates structures below the original surface level). Once the protrusions were formed, they were elongated from the bottom up by the irradiation (i.e. bottom-up process, which creates structures above the original surface level). This suggested that ion beam irradiation could cause a morphological change in the PTFE surface through not only a top down process but also a bottom-up process potentially. To approach the surface from inside the sample, energy must be transferred to the interior of the
PTFE instead of to the top surface, which can be achieved using a fast light ion beam. In this study, microstructures were fabricated by scanning the PTFE with a $3 \mathrm{MeV}$ focused proton beam, which had a diameter of $1 \mu \mathrm{m}$ on the surface. In the precedent studies, the micro-structures were formed by chemical etching [6-8] in contrast to the method based on the direct irradiation with a proton beam proposed in this study. The irradiated surfaces were observed by scanning electron microscopy (SEM).

\section{EXPERIMENTAL}

PTFE sheets (Nichias Corp.), these were $100 \mu \mathrm{m}, 300$ $\mu \mathrm{m}$, and $500 \mu \mathrm{m}$ in thickness, were used. Before irradiation, the sheets were cleaned with ethanol and dried

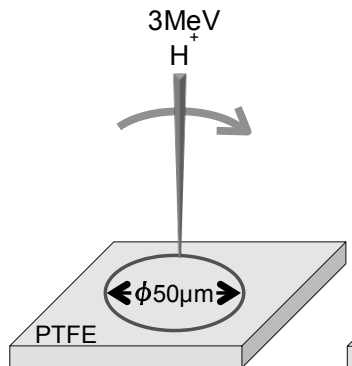

(a) Circular scanning

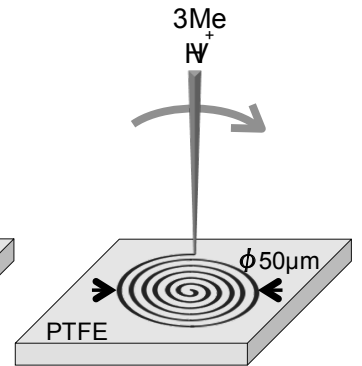

(b) Spiral scanning
Fig. 1 Scanning patterns of the proton micro beam. 

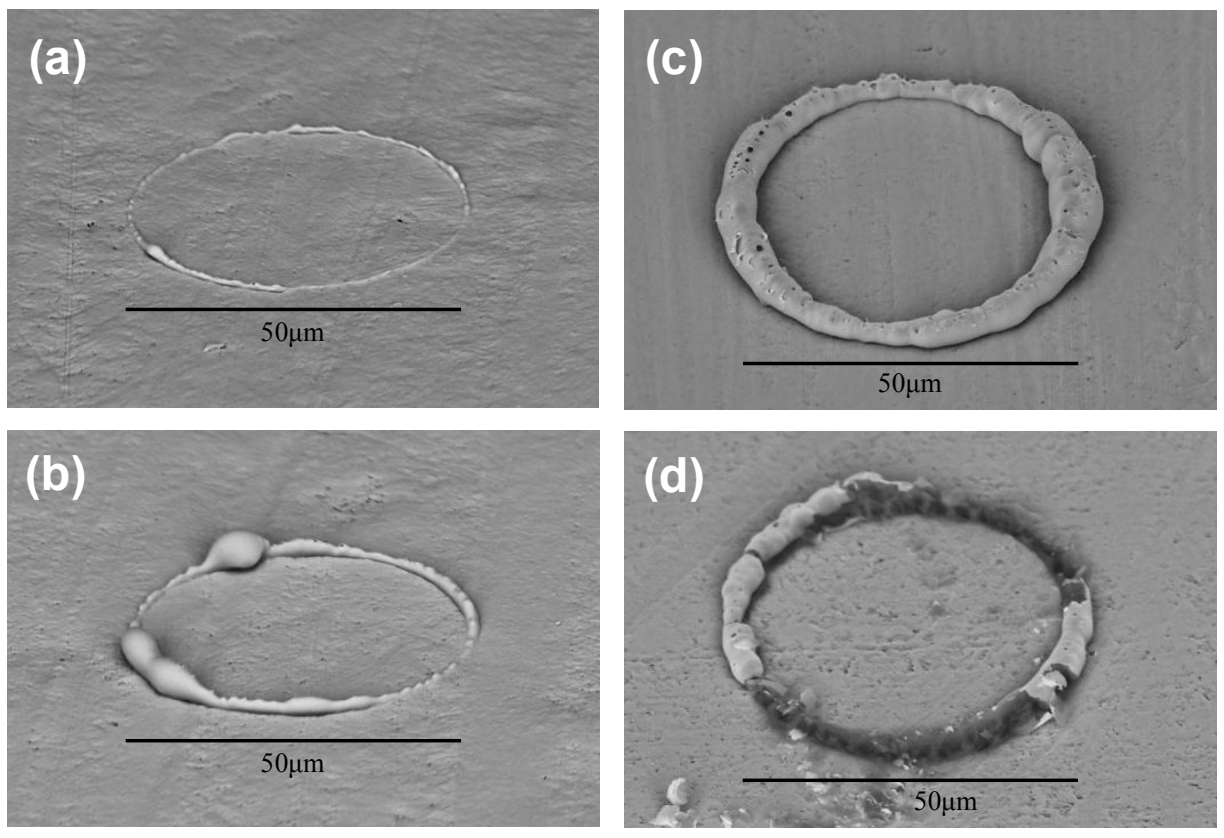

Fig. 2 SEM images of $100-\mu \mathrm{m}$-thick PTFE surfaces after proton beam scanning along a circle $50 \mu \mathrm{m}$ in diameter, after (a) 1 , (b) 5 , (c) 10 , and (d) 50 scan cycles.

at room temperature. A $3 \mathrm{MeV}$ proton beam irradiation was performed at Takasaki Ion Accelerators for Advanced Radiation Application (TIARA), JAEA. The proton beam had a diameter of $1 \mu \mathrm{m}$ on the surface of the samples, and was scanned by an electrostatic deflection scanner without a beam blanking system. The scan rate was $40 \mu \mathrm{m} / \mathrm{s}$ and the beam current was $200 \mathrm{pA}$. The beams were scanned in two scan patterns (Fig. 1). The first was a circle $50 \mu \mathrm{m}$ in diameter, where the beam repeatedly scanned the circle (Fig. 1(a)). The second was a spiral pattern, where the beam scanned once from the center of a circle $50 \mu \mathrm{m}$ in diameter (Fig. 1(b)). The surface morphology of the samples was observed by SEM (TM3000, Hitachi High-Tech. Corp.) after the surfaces were coated with a layer of gold using a plasma coater (JFC-1600, JEOL).

\section{RESULTS AND DISCUSSION}

SEM images of the circularly scanned PTFE surfaces of the 100- $\mu$ m-thick samples are shown in Fig. 2. After only one scan cycle, the width of the structure that appeared was about $1 \mu \mathrm{m}$ (Fig. 2(a)); the width was similar to the diameter of the beam spot. After 10 scan cycles, the scanned area expanded perpendicular to the original surface and partially swelled (Fig. 2(a)-(c)). In addition, the structure was rounded and a number of microholes had formed at the top (Fig. 2(c)). Because the process of expansion was inhomogeneous, the height and the width ranged from 2 to $5 \mu \mathrm{m}$. After 50 cycles, the expanded structures broke up because of overscanning, which indicated that the swelling of scanned area had peaked at some time during the 50 cycles (Fig. 2(d)).
Figure 3 shows SEM images of the surfaces of the PTFE samples of $300 \mu \mathrm{m}$ and $500 \mu \mathrm{m}$ in thickness after 10 cycles of circular scanning. In the $300-\mu \mathrm{m}$-thick sample, an expanded structure containing many needles and nanoholes was formed on the top surface of the structure, which was not observed in the $100-\mu \mathrm{m}$-thick
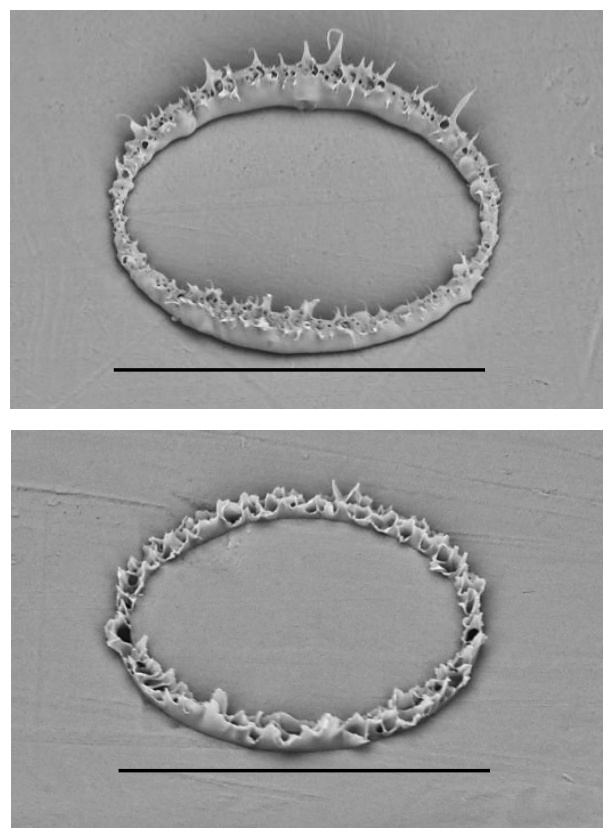

Fig. 3 SEM images of the PTFE surfaces after 10 cycles of proton beam scanning along a circle $50 \mu \mathrm{m}$ in diameter, for the (a) 300- $\mu \mathrm{m}-$ and (b) $500-\mu \mathrm{m}$-thick samples. 

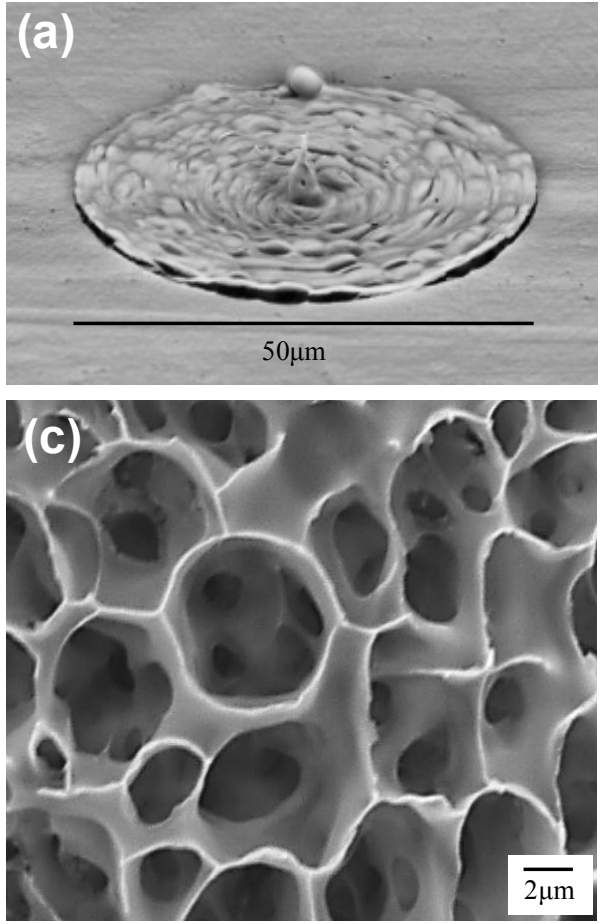

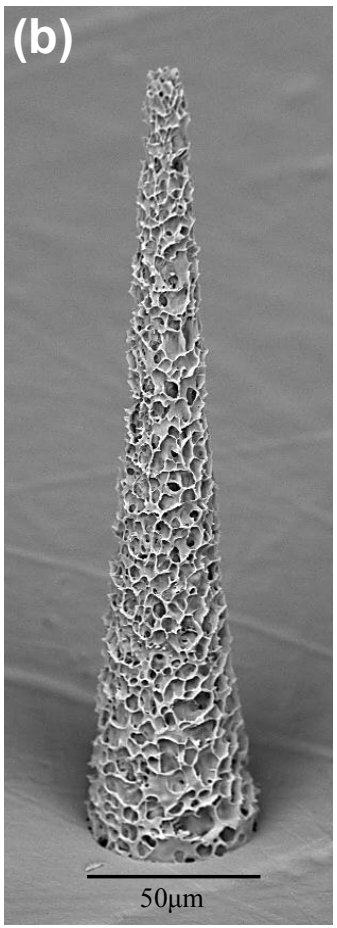

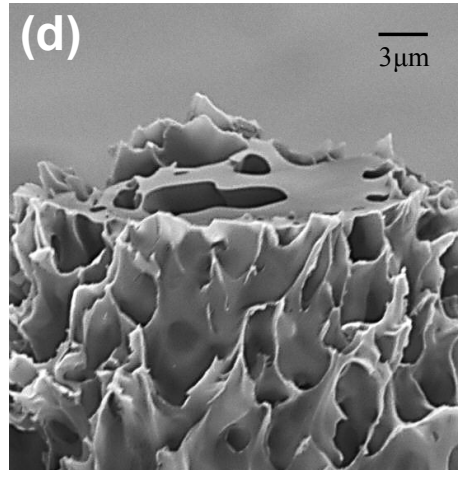

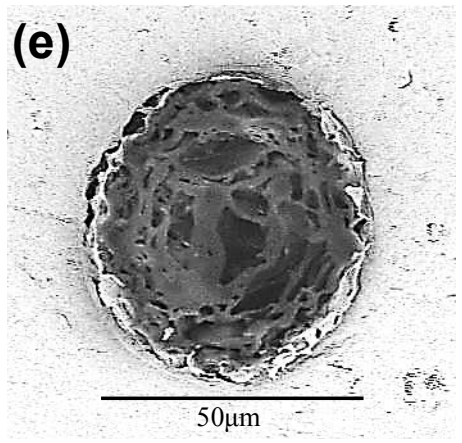

Fig. 4 SEM images of PTFE surfaces after spiral scanning from the center, for the (a) $100-\mu \mathrm{m}$ - and (b) $500-\mu \mathrm{m}$-thick samples. (c) Enlarged SEM image of the surface of the cone in Fig. 4(b). (d), (e) SEM images of the cross sections of the top and bottom of the cone, respectively.

sample (Fig. 3(a)). In the 500- $\mu \mathrm{m}$-thick sample, the scanned area did not expand and became rough with many jagged holes that were arranged in a circle of 50 $\mu \mathrm{m}$ in diameter (Fig. 3(b)). Comparison of the structures shown in Fig. 1(c) and Fig. 3 revealed that the structures gradually became rougher as the sample thickness increased.

Figure 4 shows SEM images of the PTFE surface after spiral-scanning. The irradiated area in the $100-\mu \mathrm{m}$-thick sample was lumpy, a conical projection appeared at the center, and cracks formed at the boundary between the scanned and un-scanned area (Fig. 4(a)). In the 500- $\mu$ m-thick sample, a circular cone around $250 \mu \mathrm{m}$ high, covered with jagged holes was formed (Fig. 4(b) and (c)). Figure 4(d) and 4(e) show the cross sections of the top and bottom of the cone, respectively, and indicate that the cone was porous. Figure 4(e) shows that the base of the cone was $50 \mu \mathrm{m}$ in diameter, which corresponds to the scan area.

Figure 5 shows our proposed mechanism for the morphological change in the PTFE surface induced by proton microbeam irradiation. When the PTFE surface was exposed to a focused $3 \mathrm{MeV}$ proton beam, the protons traveled along a straight line with some transverse fluctuation [6]. The range of the protons is approximately $88 \mu \mathrm{m}$ in PTFE according to the Stopping and Range of Ions in Matter calculation, and the protons transferred their energy in a sharp peak toward the end of their range, which is known as a Bragg peak (Fig. $5(a))$. Gases were generated by main-chain scissions of the PTFE molecules near the Bragg peak (Fig. 5(b)), and the temperature along the proton trajectories was elevated which softened the PTFE. In this process, the volume along the ion trajectories expanded (Fig. 5(c)), and thus pushed up the top surface (Fig. 5(d), 5(e)). The shape of the surface microstructures depended on the sample thickness.

In the $100-\mu \mathrm{m}$-thick sample, the Bragg peak was near the bottom of the sample. The direction of the expansion was both the top and the bottom of the sample, which meant that there was less pressure on the uppermost surface, and that it did not swell significantly (Fig. 5(d)). The back side surface of the sample is shown in Fig. 6. The back side of the irradiated area melted and some holes from which gas escaped were formed. In contrast, in the $500-\mu \mathrm{m}$-thick sample the direction of the expansion was only the top surface since the surface of the bottom did not change (Fig. 5(e)). Because the microbeam scanned spirally from the center, the volume expansion began with the irradiation and the cone was formed. When the generated gasses were escaped with the volume expansion, the surface of the corn became porous as shown in Fig. 4(c). 

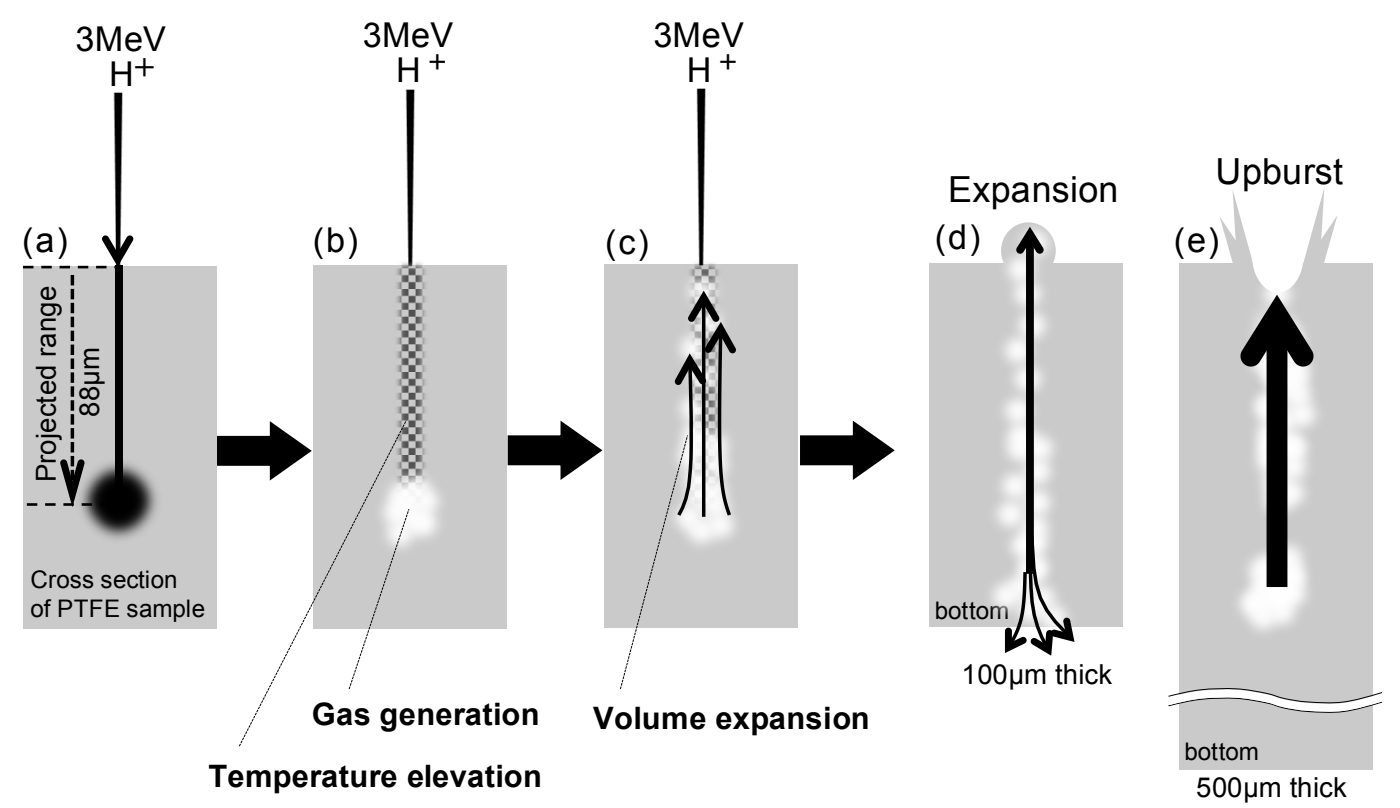

Fig. 5 Mechanism for the morphological changes in the PTFE surface induced by proton micro beam irradiation.

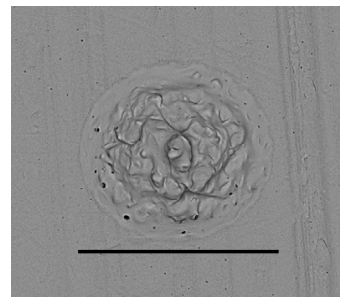

Fig. 6 SEM images of back sides of PTFE surfaces after spiral scanning from the center for the $100-\mu \mathrm{m}$-thick samples.

\section{CONCLUSION}

We have fabricated an independent microstructure on a PTFE surface using a $\mathrm{MeV}$ proton beam. The proton beam, which was $1 \mu \mathrm{m}$ in diameter on the surface, was scanned in either a circle or a spiral. The scanned areas were raised from inside the sample, which formed the surface structures. This morphological change was caused by the volume expansion due to both the temperature elevation and the gas generation along the proton trajectories inside the PTFE samples. The shape of the structures depended on the thickness of PTFE samples. A circular cone was formed by the spiral scanning of the proton beam from the center. We can therefore conclude that $\mathrm{MeV}$ proton beam irradiation is a useful method for creating independent microstructures on PTFE surfaces via a bottom-up process.

\author{
5. REFERENCES \\ [1] A. Oshima, Y. Tabata, et. al., Radiat. Phys. Chem., \\ 45, 269-273 (1995). \\ [2] N. Miyoshi, A. Oshima, et. al., Radiat. Phys. Chem., \\ 80, 230-235 (2011). \\ [3] A. Kitamura, T. Kobayashi, T. Meguro, A. Suzuki, T. \\ Terai, Trans. Mater. Res. Soc. Jpn., 33, 4, 1035-1038 \\ (2008). \\ [4] A. Kitamura, T. Kobayashi, T. Meguro, A. Suzuki, T. \\ Terai, Surf. Coat. Technol., 203, 17-18, 2406-2409 \\ (2009). \\ [5] A. Kitamura, T. Kobayashi, A. Suzuki, T. Terai, Surf. \\ Coat. Technol., 206, 841-844 (2011). \\ [6] F. Watt, M. B. H. Breese, A. A. Bettiol, J. A. van \\ Kan, materialstoday, 6, 10, 20-29 (2007). \\ [7] N. Uchiya, T. Harada, M. Murai, H. Nishikawa, J. \\ Haga, T. Sato, Y. Ishii, T. Kamiya, Nucl. Inst. Meth., B \\ 260, 405-408(2007). \\ [8] Y. Furuta, H. Nishikawa, T. Satoh, Y. Ishii, T. \\ Kamiya, R. Nakao, S. Uchida, Nucl. Inst. Meth., B 267, \\ 2285-2288 (2009).
}

(Received January 20, 2012; Accepted May 3, 2012) 